\title{
Competencias y formación universitaria del educador deportivo en Italia
}

\author{
Competences and college training of sports educators in Italy
}

\author{
Competências e treinamento faculdade educador desportivo em Itália
}

\author{
Claudia Maulini, ${ }^{a}$ Antonio Fraile Aranda, ${ }^{b}$ Rufino Cano. ${ }^{c}$ \\ a Università degli Studi di Roma “Foro Itálico”, Italia. Fono: 0636733379. \\ Correo electrónico: claudia.maulini@uniroma4.it \\ b Universidad de Valladolid, España. Fono: 983343957. Correo electrónico: afraile@mpc.uva.es \\ cUniversidad de Valladolid, España. Fono: 983183834. Correo electrónico: rcano@pdg.uva.es
}

\begin{abstract}
RESUMEN
La finalidad de este artículo es comprobar si las competencias de los programas de formación de algunas Facultades de Ciencias Motoras, en Italia, cubren las necesidades de los profesionales deportivos para atender las actividades físicas, recreativas y deportivas de los ciudadanos. Para ello, hemos desarrollado una metodología mixta desde instrumentos cualitativos y cuantitativos, como la entrevista semiestructurada y el cuestionario. Se entrevistó a 20 entrenadores y 3 responsables de proyectos de intervención educativa, cuyos resultados nos han evidenciado las competencias educativas fundamentales para dicha actividad profesional. Posteriormente, hemos diseñado un cuestionario aplicado a los estudiantes de $3^{\circ}$ curso de tres Facultades de Ciencias Motoras italianas para conocer su grado de valoración de las competencias educativas que poseen los profesionales deportivos y cómo han sido adquiridas. Los entrevistados destacan 29 competencias fundamentales para el educador deportivo. La mayoría de ellos $(84,4 \%)$ considera que posee dichas competencias.
\end{abstract}

Palabras clave: deporte, educador deportivo, competencias educativas, formación universitaria de Ciencias Motoras.

\begin{abstract}
The aim of this article has been to identify the educational competences able to ensure that sport is truly an educational tool aimed at developing the well-being among youth. For this we have detected the competences that coaches and those who are responsible of educational projects think to be fundamental for their profession and if the same competences are usually developed in the curricula of the sport sciences department of Latium Region in Italy. The methodology used has been mixed, qualitative and quantitative, using data collection techniques such as semi-structured interviews and questionnaires. In the first step 20 sports coaches were interviewed and, 2 people responsible, in Italy, of two educational projects focused on sport and social intervention. The results of the interviews allowed us to construct a questionnaire that was submitted to the students of the third year of the Bachelor's degree course in physical education and sports. The results of the quantitative phase of this research have shown that most students consider as essential, to their future work, the 29 educational competences arising from the content analysis of the interviews that have been analysed in the qualitative phase. The data show that $84.4 \%$ of the students interviewed have declared to possess the educational competences but that these have been acquired outside university.
\end{abstract} Sciences.

Key words: sport, sports educator, educational competences, university education in Motor and Sport 


\begin{abstract}
RESUMO
A finalidade de este artigo é comprovar se as competências dos programas de formação de algumas faculdades de Educação Física na Itália cobrem as necessidades dos profissionais da área de modo a atender às atividades físicas, recreativas e desportivas dos cidadãos. Para tal, desenvolvemos uma metodologia mista, incluindo instrumentos qualitativos e quantitativos, como a entrevista semiestruturada e o questionário. Foram entrevistados 20 treinadores e três responsáveis de projetos de intervenção educativa; resultados permitiram evidenciar as competências educativas fundamentais para a referida atividade profissional. Posteriormente, desenvolvemos um questionário para ser aplicado aos estudantes do $3^{\circ}$ ano de três faculdades italianas de Educação Física, visando conhecer o seu grau de valoração das competências educativas que os profissionais de esta área devem possuir e de que modo elas são adquiridas. Os entrevistados destacaram 29 competências fundamentais para o educador desportivo. A maioria de eles $(84,4 \%)$ considera que possui tais competências.
\end{abstract} Física.

Palavras chave: esporte, educador desportivo, competências educativas, formação universitária de Educação

\title{
1. INTRODUCCIÓN
}

Los cambios sociales relevantes que han tenido lugar en las últimas décadas (Bauman, 2003, 2002) han llevado a la manifestación y aparición de formas de malestar cada vez más complejas en los jóvenes (Miatto, 2012), que han puesto en evidencia la necesidad de intervenciones socio-pedagógicas dirigidas más específicamente a la persona (Morin, 2000a, 2000b, 1993). De hecho, además de las distintas formas de malestar, se han desarrollado otras denominadas "malestar de la normalidad", ligadas a las relaciones sociales y familiares, cada vez más "líquidas" e inestables, que crean graves evidencias de desorientación y fragilidad (Merico, 2004; Bauman, 2003, 2002).

Los jóvenes han sido los más afectados y sensibles a estos cambios, sobre todo, en términos de valores. La crisis de los tradicionales agentes educativos y de socialización (familia, escuela, Iglesia) ha conducido a la sociedad hacia una falta de referentes educativos. El malestar juvenil se manifiesta, en la mayoría de los casos, a través de comportamientos que contrastan con los valores sobre los que se fundamenta la vida de la comunidad y de la sociedad, como son: la hiposocialización, la agresividad, la falta de esfuerzo, la falta de respeto hacia el otro y hacia las reglas de convivencia civil, la intolerancia, la ausencia de motivación, el uso de sustancias estupefacientes, etc. (Pietropolli, 2008).

El deporte, en cuanto actividad cultural y educativa, de tipo no formal e informal, y como "agencia" de socialización alternativa, debe estar dotado de una auténtica intencionalidad dirigida a la mejora y al crecimiento de la persona. Debiese, entonces, responder a este vacío de valores como instrumento de promoción del bienestar y de la integración social, desarrollando, al mismo tiempo, una función subsidiaria con respecto a un macrosistema socio-educativo (Maulini, 2012, 2011, 2006a; Isidori e Fraile, 2008; Diener, Oishi \& Lucas, 2003; Helliwell, 2002).

Algunas investigaciones relativas a las funciones del educador deportivo (Pascual Baños, 2011; Isidori, 2009a, 2009b; Davi, 2008; Jones, Armour \& Potrac, 2004) han evidenciado cómo es posible construir una verdadera pedagogía social basada en el deporte. No sólo porque éste es capaz de incidir sobre las vivencias de las personas y de generar emociones que motiven profundamente a los sujetos en un empeño, sino, también, por la capacidad de posibilitar un cambio y una mejora de la sociedad (Petitpas, Cornelius, Van Raalte \& Jones, 2005; Damon, 2004; Larson, 2000). 
En Italia, así como en algunos países del entorno mediterráneo, como es el caso de España, precisamos de estudios pedagógicos específicos que nos ayuden a superar el malestar juvenil a través del deporte, así como de proyectos de intervención promovidos por entidades y agentes educativos a partir de un análisis científico de tipo socio-pedagógico con instrumentos metodológicamente válidos. Se trata de propiciar prácticas de intervención científicas, críticas y reflexivas que favorezcan la expresión del potencial educativo del deporte y que incidan en el ámbito social.

Se pone en evidencia, pues, la importancia de una formación psicopedagógica, y no sólo técnica, dirigida a los profesionales del deporte. Esto significa que es fundamental desarrollar en el entrenador competencias educativas que le permitan no ser solamente un técnico, responsable de la preparación física y táctica de los jugadores, sino, sobre todo, un educador consciente, capaz de utilizar el deporte para transmitir valores, favorecer el aprendizaje de competencias para la vida y, a la vez, promover procesos de empoderamiento (Maulini, 2012, 2011, 2006a; Jones et al., 2004) a través de una relación que sea intencionalmente educativa y capaz de construir procesos de crecimiento y de bienestar social. El desarrollo de tales competencias, que permitan hacer comprender el potencial educativo del deporte e incidan en la superación del malestar social, sólo será posible mediante un sistema de formación integrado que implique a la universidad, a la escuela, a la familia y a las entidades locales y de promoción del deporte (Isidori e Fraile, 2008; Maulini, 2006a; Brandani e Tomisich, 2005).

\section{METODOLOGÍA}

A partir de la necesidad de comprobar si las competencias que recogen los programas de formación de algunas Facultades de Ciencias Motoras de Italia cubren las necesidades de los profesionales deportivos para atender las actividades físicas, recreativas y deportivas de los ciudadanos, hemos establecido tres objetivos específicos:

a) Identificar las competencias educativas necesarias para asegurar que el deporte sea, verdaderamente, un instrumento educativo y promotor junto con otros agentes educativos del bienestar de los jóvenes.

b) Conocer el grado de importancia que los estudiantes de las Facultades de Ciencias Motoras de la Región del Lazio otorgan a las competencias educativas que, necesariamente, deben poseer y desarrollar los profesionales deportivos.

c) Verificar, a partir de la percepción de los estudiantes de las Facultades de Ciencias Motoras de la Región del Lazio, si estas competencias educativas son desarrolladas, o no, en el currículo universitario.

Para alcanzar dichos objetivos hemos empleado, complementariamente, una metodología cualitativa/cuantitativa, ya que el proceso de investigación, desde la aplicación de ambos paradigmas, ha de aportarnos resultados relevantes de acuerdo con los objetivos inicialmente establecidos. A este respecto, hemos de considerar que ninguno de los enfoques es mejor que el otro, sino que se trata de diferentes aproximaciones al estudio de un fenómeno. En el mismo sentido, pensamos que ni la investigación cualitativa ni la cuantitativa se pueden caracterizar por la elección de unos métodos por encima de otros (Flick, 2007). Aunque sí es verdad que cada una de ellas presupone una forma de enfocar el problema objeto de estudio, así como la decisión de elegir unos u otros instrumentos para la recogida de los datos. 
El enfoque cualitativo nos ha permitido responder al primer objetivo de investigación, además de poder llegar a contrastar, matizar y ajustar los conceptos a tener en cuenta para consolidar las variables más relevantes de nuestra investigación. Finalmente, nos ha servido para complementar la interpretación y discusión de los resultados cuantitativos.

Para identificar las competencias educativas que los entrenadores o profesionales deportivos necesitan desarrollar para promover el bienestar en los jóvenes a través del deporte, hemos utilizado la técnica de la entrevista semi-estructurada. Asimismo, para garantizar su validez nos hemos servido del juicio crítico de un grupo de expertos, con el objeto de verificar y compartir su estructura y exhaustividad y, al mismo tiempo, establecer un buen grado de coherencia con los objetivos de la investigación. Además, para mejorar la fiabilidad de los resultados obtenidos con dicha técnica, hemos requerido, de nuevo, de la intervención de un grupo de expertos, con quienes hemos coincidido en agrupar las expresiones recogidas en una misma categoría (Guba, 1981). Finalmente, hemos triangulado los datos obtenidos a través de las diferentes fuentes, con el fin de depurar la información y asegurar que los resultados tratados de forma interconectada son fiables (Patton, 2001; Goetz \& Lecompte, 1988; Guba, 1981). La muestra participante en la fase cualitativa de esta investigación está formada por 20 entrenadores y 3 responsables de los proyectos de intervención educativa a través del Rugby.

En esta primera fase, partiendo de la categoría general "Competencia educativa del entrenador", hemos analizado 3 subcategorías, divididas, a su vez, en 29 indicadores, tal y como se puede apreciar en la siguiente tabla.

Estos indicadores analizados han sido utilizados como variables de estudio en la fase cuantitativa. La finalidad de esta fase es cumplir el segundo y el tercer objetivo de esta investigación. La utilización de un cuestionario "ad hoc" nos ha permitido analizar el grado de valoración que aportan los futuros profesionales deportivos a los diferentes elementos que constituyen la competencia educativa de sus homólogos deportivos con experiencia en la práctica, así como averiguar si han adquirido, o no, dichas competencias mediante la formación universitaria que recibieron en su momento o, por el contrario, si las adquirieron fuera de este contexto académico.

Para validar el contenido de los cuestionarios se han empleado dos procedimientos: por un lado, el juicio crítico emitido por un grupo de expertos; por otro, un test preliminar a un grupo piloto. A su vez, para constatar la fiabilidad, hemos utilizado el coeficiente Alfa de Cronbach, apreciando que los valores obtenidos superan el 0.895 .

La población del estudio cuantitativo ha estado formada por estudiantes del $3^{\circ}$ curso del Grado en Actividades Motoras y Deportivas de las Facultades de Ciencias Motoras de la Región de Lazio (Universidad Foro Itálico de Roma, Universidad Tor Vergata de Roma, Universidad de Cassino). El número total de estudiantes ha sido de 548 y la muestra calculada por cuotas (manteniendo la misma proporción de estudiantes por Facultad) ha sido de 179 sujetos, 136 hombres $(76,4 \%)$ y 43 mujeres $(23,6 \%)$ con una edad media de 23 años. El $47 \%$ de la muestra ya viene trabajando en el sector deportivo. Más concretamente, el $68 \%$ dirige actividades deportivas: el $41 \%$ con escolares y el $27 \%$ con adolescentes. Esto indica que ese $47 \%$ de la muestra ha respondido desde su propia experiencia profesional, y no solamente a partir de los aprendizajes adquiridos en su formación universitaria.

El análisis de los datos cualitativos ha sido elaborado a partir de la trascripción y la codificación de las informaciones obtenidas de las entrevistas. Para su codificación fue necesario, en primer lugar, definir el universo de trabajo, las unidades de análisis y sus 
Tabla $N^{\circ} 1$. Sistema de categorías, subcategorías e indicadores para el análisis de los datos cualitativos

\begin{tabular}{|c|c|c|}
\hline $\begin{array}{c}\text { Categoría teórica } \\
\text { general }\end{array}$ & Subcategorías & Indicadores \\
\hline \multirow[t]{10}{*}{$\begin{array}{l}\text { Competencia } \\
\text { educativa } \\
\text { del entrenador }\end{array}$} & \multirow[t]{3}{*}{$\begin{array}{l}\text { a) Habilidades } \\
\text { interpersonales }\end{array}$} & $\begin{array}{l}\text { a.1) Comunicar eficazmente } \\
\text { a.2) Escuchar activamente } \\
\text { a.3) Crear un clima positivo }\end{array}$ \\
\hline & & $\begin{array}{l}\text { a.4) Dialogar } \\
\text { a.5) Comprender las necesidades de los deportistas }\end{array}$ \\
\hline & & $\begin{array}{l}\text { a.6) Poseer inteligencia emocional } \\
\text { a.7) Trabajar en equipo }\end{array}$ \\
\hline & \multirow[t]{3}{*}{$\begin{array}{l}\text { b) Estrategias } \\
\text { didácticas }\end{array}$} & $\begin{array}{l}\text { b.1) Favorecer el liderazgo } \\
\text { b.2) Gestionar los errores } \\
\text { b.3) Gestionar las dinámicas de grupo } \\
\text { b.4) Compartir los objetivos }\end{array}$ \\
\hline & & $\begin{array}{l}\text { b.5) Favorecer la participación } \\
\text { b.6) Motivar a los deportistas } \\
\text { b.7) Proponer metas realizables }\end{array}$ \\
\hline & & $\begin{array}{l}\text { b.8) Trabajar en grupo } \\
\text { b.9) Favorecer la gestión de las emociones } \\
\text { b.10) Favorecer proceso de fortalecimiento } \\
\text { b.11) Favorecer el proceso de coping } \\
\text { b.12) Enseñar a través del juego }\end{array}$ \\
\hline & \multirow{3}{*}{$\begin{array}{l}\text { c) Valores de } \\
\text { referencia }\end{array}$} & c.1) Respeto \\
\hline & & $\begin{array}{l}\text { c.2) Honestidad } \\
\text { c.3) Lealtad } \\
\text { c.4) Competición }\end{array}$ \\
\hline & & $\begin{array}{l}\text { c.5) Responsabilidad } \\
\text { c.6) Diversidad } \\
\text { c.7) Amistad } \\
\text { c.8) Solidaridad }\end{array}$ \\
\hline & $\begin{array}{l}\text { d) Conocimiento de la } \\
\text { disciplina deportiva }\end{array}$ & $\begin{array}{l}\text { d.1) Técnica deportiva } \\
\text { d.2) Táctica deportiva }\end{array}$ \\
\hline
\end{tabular}

Fuente: elaboración propia.

categorías. En el sistema de categorías, la categoría teórica general "Competencia educativa del entrenador" ha sido subdividida por el grupo de expertos en cuatros subcategorías: habilidades interpersonales, estrategias didácticas, valores de referencia y conocimiento de la disciplina deportiva. Por su parte, el análisis de los datos cuantitativos se ha llevado a cabo mediante un análisis descriptivo de los datos destacados en el cuestionario a partir del paquete estadístico SPSS 15.0. 


\section{RESULTADOS}

De estos resultados se destaca que algunas competencias han sido evidenciadas, bien por los entrenadores, bien por los responsables de la Intervención Educativa a través del Deporte (IED). En la tabla siguiente, y teniendo en cuenta las cuatro subcategorías, se pueden observar tanto los indicadores destacados por los entrenadores o los responsables del IED, como aquellos otros valorados por ambos.

Tabla $N^{\circ} 2$. Competencias surgidas en las entrevistas

\begin{tabular}{|c|c|c|c|}
\hline \begin{tabular}{|c|} 
Categoría \\
teórica general
\end{tabular} & Subcategorías & Indicadores & \\
\hline \multirow[t]{10}{*}{$\begin{array}{l}\text { Competencia } \\
\text { educativa } \\
\text { del entrenador }\end{array}$} & \multirow[t]{3}{*}{$\begin{array}{l}\text { a) Habilidades } \\
\text { interpersonales }\end{array}$} & $\begin{array}{l}\text { a.1) Comunicar eficazmente } \\
\text { a.2) Escuchar activamente } \\
\text { a.3) Crear un clima positivo }\end{array}$ & $\begin{array}{l}\text { Entrenadores + } \\
\text { Responsables IED }\end{array}$ \\
\hline & & $\begin{array}{l}\text { a.4) Dialogar } \\
\text { a.5) Comprender las necesidades de los } \\
\text { deportistas }\end{array}$ & Entrenadores \\
\hline & & $\begin{array}{l}\text { a.6) Poseer inteligencia emocional } \\
\text { a.7) Trabajar en equipo }\end{array}$ & Responsables IED \\
\hline & \multirow[t]{3}{*}{$\begin{array}{l}\text { b) Estrategias } \\
\text { didácticas }\end{array}$} & $\begin{array}{l}\text { b.1) Favorecer el liderazgo } \\
\text { b.2) Gestionar los errores } \\
\text { b.3) Gestionar las dinámicas de grupo } \\
\text { b.4) Compartir los objetivos }\end{array}$ & $\begin{array}{l}\text { Entrenadores + } \\
\text { Responsables IED }\end{array}$ \\
\hline & & $\begin{array}{l}\text { b.5) Favorecer la participación } \\
\text { b.6) Motivar a los deportistas } \\
\text { b.7) Proponer metas realizables }\end{array}$ & Entrenadores \\
\hline & & $\begin{array}{l}\text { b.8) Trabajar en grupo } \\
\text { b.9) Favorecer la gestión de las } \\
\quad \text { emociones } \\
\text { b.10) Favorecer el proceso de } \\
\text { fortalecimiento } \\
\text { b.11) Favorecer el proceso de coping } \\
\text { b.12) Enseñar a través del juego }\end{array}$ & Responsables IED \\
\hline & \multirow[t]{3}{*}{$\begin{array}{l}\text { c) Valores de } \\
\text { referencia }\end{array}$} & c.1) Respeto & $\begin{array}{l}\text { Entrenadores + } \\
\text { Responsables IED }\end{array}$ \\
\hline & & $\begin{array}{l}\text { c.2) Honestidad } \\
\text { c.3) Lealtad } \\
\text { c.4) Competición }\end{array}$ & Entrenadores \\
\hline & & $\begin{array}{l}\text { c.5) Responsabilidad } \\
\text { c.6) Diversidad } \\
\text { c.7) Amistad } \\
\text { c.8) Solidaridad }\end{array}$ & Responsables IED \\
\hline & $\begin{array}{l}\text { d) Conocimiento } \\
\text { de la disciplina } \\
\text { deportiva }\end{array}$ & $\begin{array}{l}\text { d.1) Técnica deportiva } \\
\text { d.2) Táctica deportiva }\end{array}$ & Entrenadores \\
\hline
\end{tabular}

Fuente: elaboración propia. 
Respecto a la subcategoría conocimiento de la disciplina deportiva, sólo los entrenadores han subrayado la importancia de los aspectos técnicos y tácticos. Esto es así, probablemente, porque los entrenadores y los educadores tienen finalidades diferentes en sus respectivas actividades como enseñantes. En las intervenciones educativas, el deporte representa el medio para alcanzar las finalidades primarias, que tienen que ver con la intención de favorecer el bienestar en los jóvenes y reducir formas de malestar; mientras que en los entrenadores de las diferentes disciplinas deportivas, las finalidades son, sobre todo, enseñar cómo practicar el deporte y, en la mayoría de los casos, obtener resultados y buena prestación.

De la muestra que afecta a los estudiantes de las Facultades de Ciencias Motoras de la Región de Lazio, hemos de señalar los siguientes puntos de interés:

1. Todas las competencias han obtenido una valoración positiva. Se deduce, por tanto, que la mayoría de los estudiantes consideran importantes o muy importantes las competencias indicadas en el cuestionario. Entre las competencias que han obtenido una valoración positiva, las que, finalmente, han logrado un porcentaje de respuesta más alto $(93,8 \%)$ y, en consecuencia, son consideradas en la muestra como importantes, aparecen: saber observar, saber motivar a los deportistas, saber comunicar eficazmente, y crear un clima de confianza en el grupo.

Grafico $N^{\circ} 1$. Competencias más valoradas con $\%$ de respuesta >=80\%

\section{COMPETENCIAS DEL PROFESIONAL DEL DEPORTE CONSIDERADAS "MUY IMPORTANTES" (\% DE RESPUESTA >=80\%)}

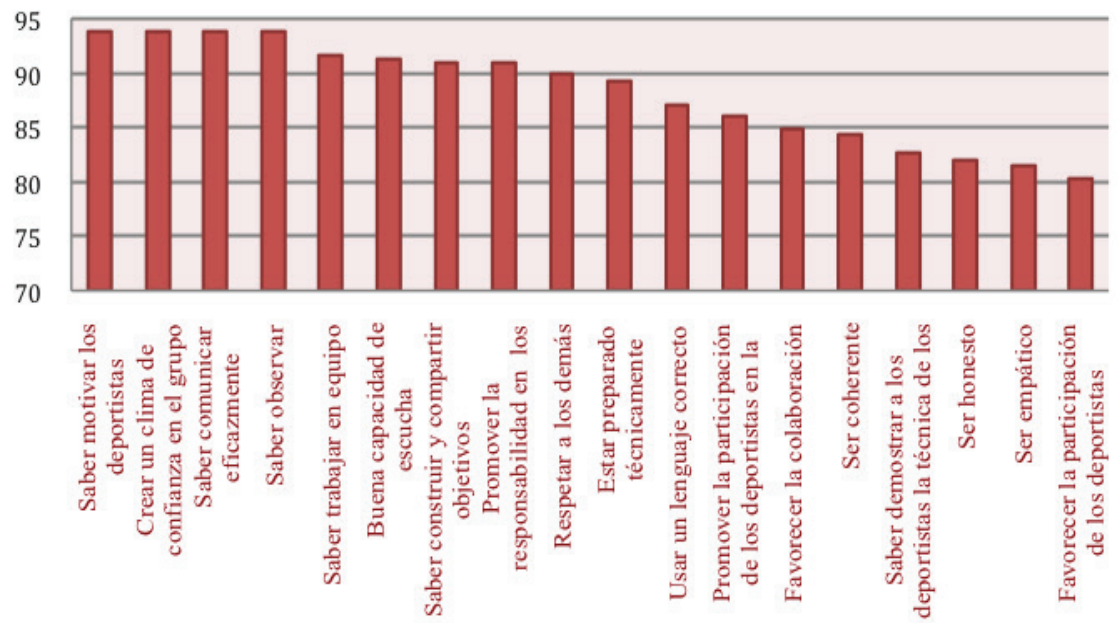

Fuente: elaboración propia.

2. Respecto a las competencias adquiridas y no adquiridas, el $84,4 \%$ de los estudiantes afirma poseer las competencias educativas necesarias para ejercer su labor profesional. En particular, se destaca que 9 de cada 10 estudiantes declaran poseer las siguientes competencias: saber observar, respetar a los demás, ser honestos, estar preparado técnicamente, tener una buena capacidad de escucha, y saber construir y compartir objetivos. Además, el 30,9\% se declara incompetente o, lo que es lo mismo, no sabe utilizar las nuevas tecnologías; el $29,2 \%$ no sabe promover la 
Estudios Pedagógicos XLI, N 1: 167-182, 2015

COMPETENCIAS Y FORMACIÓN UNIVERSITARIA DEL EDUCADOR DEPORTIVO EN ITALIA

autonomía en los deportistas, y el $24,2 \%$ no se considera un buen líder ni sabe promover la capacidad de trasladar fuera del terreno de juego lo que ha aprendido a través del deporte.

Gráfico $N^{\circ}$ 2. Competencias adquiridas y no adquiridas por los estudiantes

\section{Competencias adquiridas y no adquiridas por los estudiantes}

BUENA CAPACIDAD DE ESCUCHA

PROMOVER LA RESPONSABILIDAD EN LOS DEPORTISTAS PROMOVER LA PARTICIPACIÓN DE LOS DEPORTISTAS EN LA ORGANIZACIÓN DE LAS ACTIVIDADES

SER EMPÁTICO

PROMOVER LA CAPACIDAD DE "TRASLADAR FUERA DEI TERRENO DE JUEGO"

UTILIZAR FORMAS DE ENSEÑANZA DIFERENTES Y NUEVOS INSTRUMENTOS DIDÁCTICOS

UTILIZAR LAS NUEVAS TECNOLOGIAS SABER TRABAJAR EN EQUIPO CAPACIDAD PARA GESTIONAR Y RESOLVER LOS CONFLICTOS

SABER DEMOSTRAR A LOS DEPORTSITAS LA TÉCNICAO EJERCICIOS

CAPACIDAD PARA RESOLVER CRITICAMENTE LOS PROBLEMAS

SER CREATIVO

CREAR UN CLIMA DE CONFIANZA EN EL GRUPO

ESTAR PREPARADO TÉCNICAMENTE

SABER OBSERVAR

FAVORECER LA COLABORACIÓN

SER JUSTO

SER COHERENTE

PROMOVER LAAUTONOMIA DE LOS DEPORTISTAS

SER HONESTO

SABER COMUNICAR EFICAZMENTE

USAR UN LENGUAJE CORRECTO

SER REALISTA

SER REFLEXIVO

SER UN BUEN LÍDER

RESPETAR A LOS DEMÁS

SABER COSTRUIR/COMPARTIR OBJETIVOS

FAVORECER LA PARTICIPACIÓN DE LOS DEPORTISTAS

SER OBJETIVOS EN LAS ELECCIONES

SABER MOTIVAR A LOS DEPORTISTAS

BUENA CAPACIDAD ORGANIZATIVA

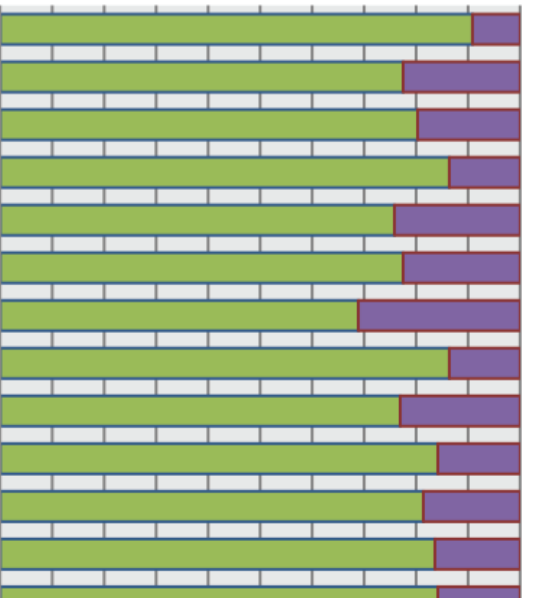

Posee $\quad \square$ posee
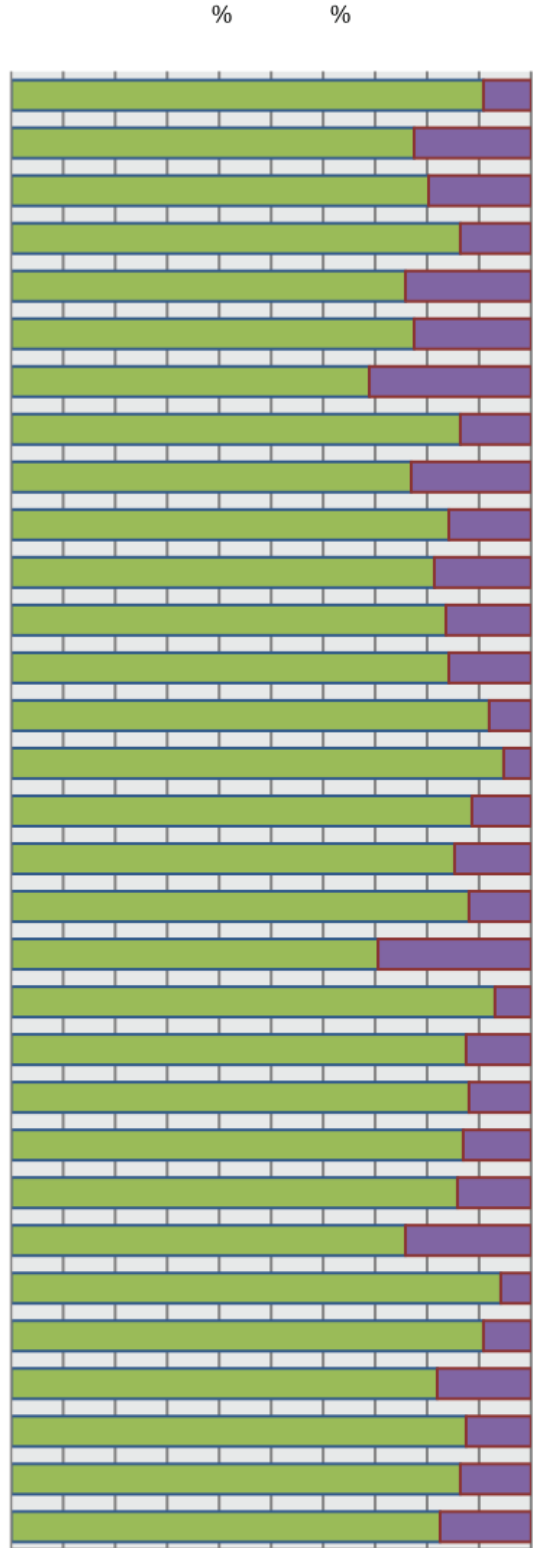

$\begin{array}{lllllllllll}0,0 & 10,0 & 20,0 & 30,0 & 40,0 & 50,0 & 60,0 & 70,0 & 80,0 & 90,0 & 100,0\end{array}$

Fuente: elaboración propia. 
3. En relación a las competencias adquiridas fuera de la formación universitaria, el análisis de los datos obtenidos revela que el $50 \%$ de los estudiantes considera haber desarrollado a lo largo de su vida (al margen de la formación universitaria), el respeto hacia los demás $(88,2 \%)$, el ser honesto $(87,6 \%)$ y el ser coherente $(83,7 \%)$.

Gráfico $N^{\circ}$ 3. Competencias desarrolladas fuera de la formación universitaria con $\%$ de respuesta $=<50 \%$

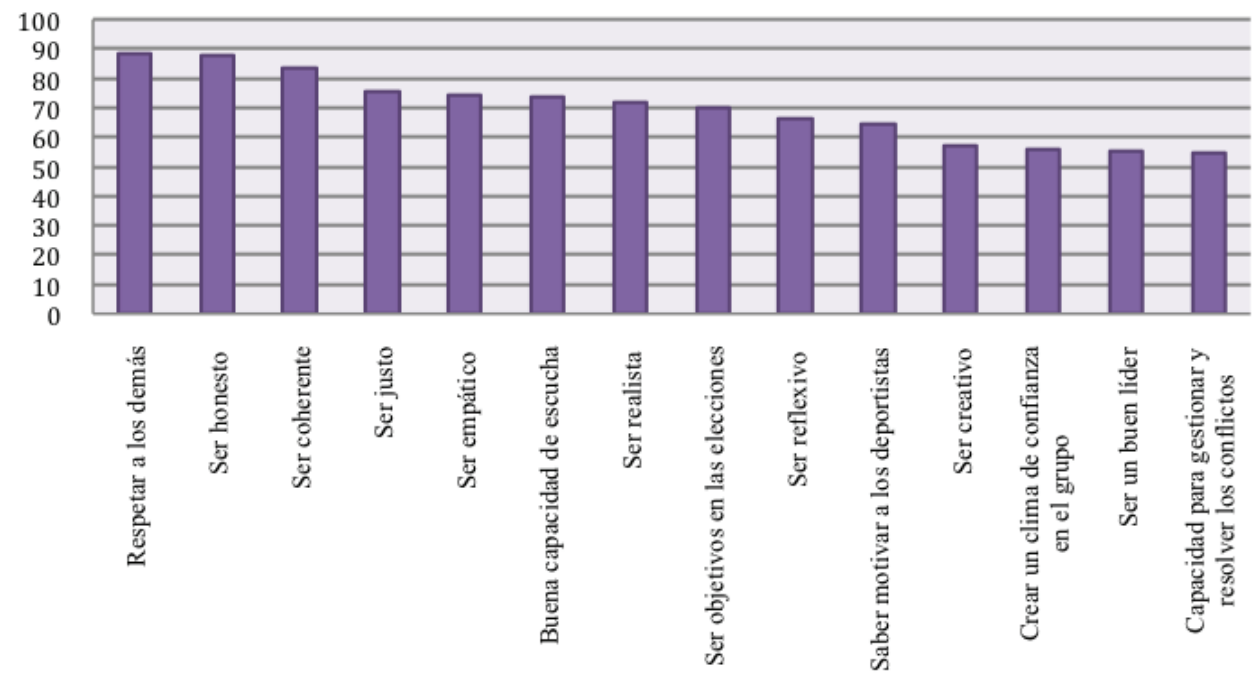

Fuente: elaboración propia.

4. En cuanto a las competencias adquiridas mediante la formación universitaria, el análisis de los datos recogidos concluye que el $50 \%$ de los estudiantes considera que posee las siguientes competencias: utilizar formas de enseñanza diferentes y nuevos instrumentos didácticos $(67,4 \%)$, estar preparado técnicamente $(65,7 \%)$, usar un lenguaje correcto $(63,5 \%)$ y saber dar/construir objetivos $(61,8 \%)$. 
Gráfico $N^{\circ}$ 4. Competencias desarrolladas mediante la formación universitaria con $\%$ de respuesta $=<50 \%$

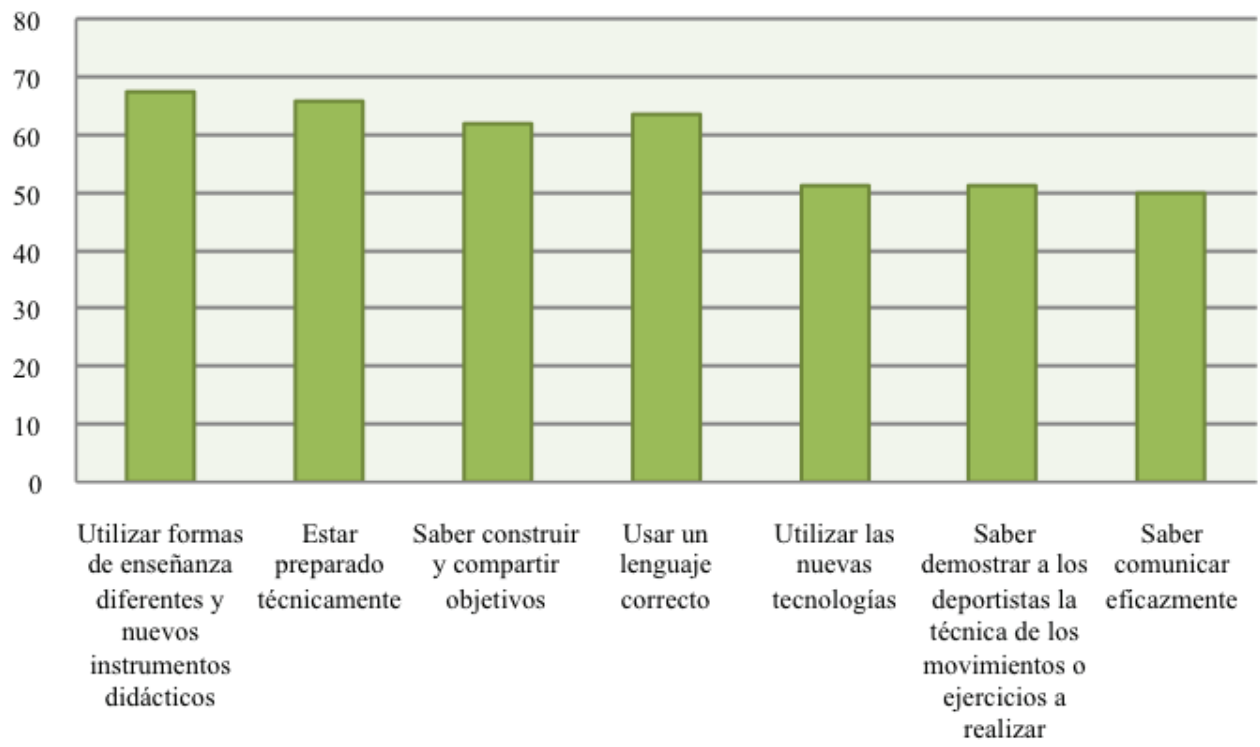

Fuente: elaboración propia.

\section{DISCUSIÓN Y CONCLUSIÓN}

Las competencias analizadas a través de las entrevistas, en la fase cualitativa, y del cuestionario, en la parte cuantitativa, nos han permitido lograr el primer objetivo del estudio. Esto, a su vez, nos ha permitido construir un sistema de competencias para el educador deportivo, hasta establecer una relación entre competencias como se destaca en la literatura (UNESCO, 2009).

Respecto a este sistema interrelacionado de competencias para los educadores deportivos, los estudiantes de $3^{\circ}$ curso del Grado en Actividades Motoras y Deportivas de las Universidades de la Región de Lazio han respondido atribuyendo un valor positivo a cada una de las competencias, lo que significa que éstas son relevantes para el profesional del deporte. Además, las competencias que destacan, tras el análisis del cuestionario, se corresponden con las que han sido seleccionadas en la bibliografía consultada. Los 20 entrenadores entrevistados, los 3 responsables de los proyectos, y el 93,8\% de los estudiantes señalan la importancia de saber motivar a los deportistas, cuestión que, como nos indican Galera (2001), Pascual Baños (2010) y Rius (2005), está estrictamente ligada a saber comunicar eficazmente y a escuchar activamente, aspectos destacados por Buber (2004), Fraile Aranda (2008), Contreras y García López (2010), así como al saber observar, tal y como revela Davi (2008).

Estas competencias permiten crear un clima positivo y de confianza en el grupo, según señala Pascual Baños (2011) en su trabajo. Asimismo, ayudan a identificar los puntos 
fuertes y débiles del deportista, además de definir unos objetivos realistas y realizables que, como ha indicado el $91 \%$ de los estudiantes, resulta de gran interés poder compartirlos con los deportistas (Maulini, 2012, 2006a; Formenti, 2006; Brandani e Tomisich, 2005; Hellison, 2003; Galera, 2001; Danish, 1998; Danish, D’Augelli \& Ginsberg, 1984).

Todo ello apunta a que el profesional del deporte debe tener un buen conocimiento de la disciplina deportiva, tanto en sus aspectos técnicos como tácticos, puesto que debe saber asociar, de una manera coherente, los objetivos y las necesidades de los deportistas tal y como han indicado los entrenadores y como se destaca en la literatura consultada (Hohmann, Lames \& Letzelter, 2005; Weineck, 2005; Thiess, Tschiene \& Nickel, 2004; Lev Pavlovich, 2001; Zhelyazkov, 2001). Estos aspectos técnico-tácticos, no obstante, no han sido destacados de manera explícita por los responsables de las intervenciones educativas analizadas, lo cual puede deberse a que durante la entrevista estaban centrados en resaltar la finalidad educativa del proyecto, apreciando el deporte, propiamente, como un medio de prevención o superación del malestar juvenil, dejando en un segundo plano la explicación de las características técnico-tácticas de dicho deporte.

Parte de la importancia de la motivación y de la búsqueda de objetivos realistas radica en que se favorece la participación de los deportistas, ya que, tal como se ha visto en la literatura (Hellison, 2003; Danish, 1998; Danish et al., 1984) y tras el estudio realizado, saber promover la participación en la actividad deportiva y en la organización de las actividades son competencias fundamentales (el $80 \%$ de los estudiantes así lo señala) para conseguir el éxito en un programa de intervención educativa a través del deporte (entrenamiento) y de las clases de Educación Física en la escuela, según plantea Maulini (2013, 2006a).

Esta importancia también viene justificada por la pirámide de las necesidades de Maslow (1973), que destaca, entre los aspectos fundamentales para el bienestar de la persona, las necesidades sociales, que implican sentirse parte de un grupo además de la posibilidad y capacidad de cooperar con los demás. Con esto se quiere responder a la necesidad de pertenencia, de participación y de aceptación, que favorecen y desarrollan la sociabilidad, la autoestima y la confianza. En consecuencia, entre los elementos que permiten el establecimiento de la relación educativa, destacamos la participación de todos los sujetos implicados en la misma, tal como indican Iori (1996) y Malavasi (2007).

Rogers (1983), en consonancia con estas cuestiones, destaca que la relación educativa que se establece entre educador y educando debiese ser aquella que se produce en un encuentro basado en el diálogo -favoreciendo una participación consciente de los actores implicados en la relación-, en el respeto y en la aceptación de la diversidad. En este sentido, el responsable de un proyecto de intervención educativa a través del Rugby subraya la relevancia de la diversidad como un valor tanto en este deporte, como en la vida en general.

Los teóricos del Positive Youth Development (Petitpas et al., 2005; Larson, 2000) subrayan la relevancia de promover la participación en actividades desarrolladas en ambientes idóneos, con vistas al crecimiento positivo de los jóvenes. Hellison (2003) y Danish (1998) indican, en sus programas de intervención educativa a través del deporte, algunas estrategias eficaces para beneficiar la participación de los jóvenes deportistas. Entre estas estrategias destaca favorecer la colaboración entre los deportistas (que el 84,8\% de los estudiantes valora como muy importante) sobre la base de trabajar en grupo, lo que se enlaza, claramente, con saber gestionar las dinámicas entre los participantes y favorecer procesos de liderazgo y de empoderamiento, promoviendo, también, la autonomía de los 
participantes, tal como aparece en las entrevistas y en la literatura (Pascual Baños, 2011; Weineberg \& Gould, 2010; Prat y Soler, 2003).

Todo lo anterior exige saber gestionar las emociones propias y de los deportistas y, por lo tanto, disponer de una buena capacidad crítica para resolver conflictos (Fraile Aranda, 2008; Goleman, 2008). Por consiguiente, que el educador deportivo sea capaz de administrar los errores de los deportistas, ayuda a que los participantes puedan desarrollar la capacidad de superación o coping, como oportunidad de crecimiento y de desarrollo de nuevas competencias (Hernández, Velázquez y Alonso, 2004; Moreno y Del Villar, 2004).

La participación implica y favorece, también, el desarrollo de valores como la honestidad (destacado por el 82\% de los estudiantes), el respeto de sí mismo y del otro (89,9\%), la solidaridad, la amistad, la lealtad, la competición (Isidori, 2009a) y la responsabilidad personal y social sobre la cual Hellison (2003) ha construido un programa de intervención educativa a través del deporte. Esta apreciación ha sido señalada por el 91\% de los estudiantes de la investigación.

La aplicación de estas competencias se destaca en las entrevistas, tanto a los entrenadores como a los responsables de los proyectos de intervención educativa, así como en el 91,6\% de los estudiantes. El saber trabajar en equipo con otros profesionales es fundamental para favorecer el desarrollo de procesos educativos sistémicos desde los cuales responder a la complejidad de la sociedad (Moreno y Del Villar, 2004).

Tras los resultados alcanzados, consideramos que todas las competencias educativas han sido valoradas de forma positiva por los entrenadores y estudiantes de las Facultades de Ciencias Motoras, confirmando las reflexiones de los estudios revisados. Igualmente, conviene recordar la importancia destacada, a través de las entrevistas y del cuestionario, de una formación integral que favorezca, además de las competencias técnico-tácticas, las competencias educativas, entendiendo por ellas las habilidades interpersonales, las estrategias didácticas y los valores de referencia.

En relación al tercer objetivo relativo al desarrollo de las competencias educativas en los estudiantes de las Facultades de Ciencias Motoras de la Región Lazio, hemos de señalar una serie de indicaciones importantes. El 84,4\% de los estudiantes, además de afirmar que poseen las competencias educativas necesarias, señalan que la mayoría ha sido adquirida a través de itinerarios formativos-experienciales extrauniversitarios. En este punto es necesario recordar, como hemos señalado anteriormente, que la muestra está constituida por un $47 \%$ de estudiantes que ya trabajaba en el sector deportivo; de ellos, el $68 \%$ trabaja en el sector juvenil (el $41 \%$ con niños y el $27 \%$ con adolescentes). Este dato es especialmente significativo porque indica que el $47 \%$ de la muestra ha contestado en base a su propia experiencia profesional, y no solamente atendiendo a lo que han aprendido en su formación universitaria. La competencia, como indica Meghnagi (2005), no es sólo el resultado exclusivo de la formación recibida en contextos formales, sino también de un saber profesional que, como ha subrayado Schön (1983), es importante valorar a través de una reflexión de las experiencias personales.

También es interesante destacar que las competencias que los estudiantes han desarrollado durante la formación universitaria corresponden, mayoritariamente, a las relacionadas con los aspectos técnico-tácticos del deporte (Isidori, 2009a), seguidas de estar preparado técnicamente (65,7\%), saber demostrar a los deportistas la técnica de los movimientos o ejercicios a realizar $(51,1 \%$ ) y utilizar formas de enseñanza diferentes y nuevos instrumentos didácticos $(67,4 \%)$, esta última desarrollada en diferentes cursos específicos 
en las Facultades de Ciencias Motoras, como Teoría y Metodología del Entrenamiento, Juegos Deportivos, etc. ${ }^{1}$, y no tanto a través de los contenidos de carácter psicopedagógico.

Por mediación de la formación universitaria recibida destaca la adquisición de competencias transversales tales como: saber formular y construir objetivos $(61,8 \%)$, usar un lenguaje correcto $(63,5 \%)$, utilizar nuevas tecnologías $(51,1 \%)$, en consonancia con las recomendaciones del Parlamento Europeo (OCDE, 2006) sobre las "Competencias clave para el aprendizaje permanente", en los "Descriptores de Dublín” (Processo di Bologna, 2004) $)^{2}$ y en el Tuning Educational Structures in Europeo (González y Wagenaar, 2008).

Nos satisface comprobar, desde planteamientos profesionales y de investigación, que estos datos concuerdan con los obtenidos en otros estudios en donde el 49,4\% de los estudiantes afirma haber desarrollado la capacidad organizativa fuera de la formación universitaria, y sólo el 33,1\% gracias a ella. Respecto a la capacidad de promover la autonomía de los deportistas, principio fundamental de la pedagogía y de la educación (Corsi, 2003; Freire, 2004), el 33,1\% de los estudiantes de las Facultades de Ciencias Motoras sostiene haberla adquirido fuera de la formación universitaria, el 37,6\% a través de la actividad académica, y un 29,2\% considera no haberla desarrollado.

Similar es la situación relativa a la promoción en el grupo de reflexión para desarrollar en los deportistas la capacidad de "trasladar fuera del terreno de juego" lo aprendido en el deporte (Hellison, 2003; Danish, 1998; Danish et al., 1984). Respecto a esta competencia, el 43,8\% ha afirmado haberla desarrollado mediante la formación universitaria, frente a un $24,2 \%$ que afirma no poseerla. Estos resultados tienen, claramente, una correspondencia con la consideración del deporte como instrumento educativo, sobre todo al constatar que muchos de los entrenadores y de los responsables de los proyectos de intervención entrevistados han señalado el valor de promover la reflexión con los deportistas.

Finalmente, en cuanto a la capacidad de promover la responsabilidad de los deportistas, el $43,8 \%$ sostiene haberla desarrollado fuera de la Universidad y un 33,7\% durante su formación universitaria. No obstante, un $22,5 \%$ de los estudiantes, lo que nos preocupa, afirma no poseerla, a pesar de la enorme importancia otorgada por Hellison (2003) y destacada por los autores de este trabajo en las entrevistas.

Es evidente, pues, que el desarrollo de las competencias educativas se atribuye, mayoritariamente, a las experiencias profesionales que los estudiantes ya poseen, denominadas know how o saber profesional. Éstas deberían ser valoradas, en mayor medida, mediante la aplicación de estrategias didácticas como las action learning (Maulini, 2006b), y profundizadas a través de un estudio universitario que respondiera a las necesidades de los estudiantes, estando expresamente presentes en los programas académicos.

Por último, podemos concluir que las competencias adquiridas por los estudiantes de $3^{\circ}$ curso del Grado en Actividades Motoras y Deportivas de las Facultades de Ciencias Motoras del Lazio en la formación universitaria son, fundamentalmente, técnico-deportivas. Esto nos lleva a afirmar que la formación de estos profesionales deportivos es, prioritariamente, de carácter técnico-deportivo, descuidándose la formación psicopedagógica y educativa que, efectivamente, permitiría superar un modelo exclusivamente técnico en la práctica educativo-deportiva.

Cfr. Guía del estudiante de la Universidad de Roma Foro Itálico (2010-2011).

Cfr. www.jointquality.org 


\section{REFERENCIAS BIBLIOGRÁFICAS}

Bauman, Z. (2003). Dentro la globalizzazione. Le conseguenze sulle persone. Roma-Bari: Laterza. (2002). Modernità liquida. Roma-Bari: Editori Laterza.

Brandani, W. e Tomisich, M. (2005). La Progettazione Educativa. Il Lavoro Sociale Nei Contesti Educativi. Roma: Carocci.

Buber, M. (2004). Il principio dialogico. Milano: San Paolo Edizioni.

Contreras, O. R. y García López, M. (2010). Comunicación y aprendizaje en Educación Física. En C. González Arévalo y T. Lleixa Arribas (Coords.), Didáctica de la educación física (pp. 47-64). Barcelona: Graò.

Corsi, M. (2003). Il coraggio di educare. Il valore della testimonianza. Milano: Vita e Pensiero.

Damon, W. (2004). What is Positive Youth Development? Annals of American Academy of Political and Social Science, vol.591 (1), 13-24.

Danish, S. J. (1998). Learning and Teaching Life Skills through Sport. II Encontro Internacional de Psicologia Aplicada ao Desporto e Actividade Fisica. Braga: Universidade do Minho.

, D’Augelli, A. R. \& Ginsberg, M. R. (1984). Life Development Intervention: Promotion of Mental Health through the Development of Competence. In S. D. Brown \& R. V. Lent (Eds.), Handbook of Counseling Psychology (pp. 520-544). New York: Wiley.

Davi, M. (2008). Padronanze trasversali nelle competenze dell'educatore. In R. Farné (Coord.), Sport e Formazione (pp. 169-190). Milano: Edizione Guerini e Associati.

Diener, E., Oishi, S. \& Lucas, R. E. (2003). Personality, Culture, and Subjective Well-Being: Emotional and Cognitive Evaluations of Life. Annual Review of Psychology, (54), 403-425.

Formenti, L. (2006). Psicomotricità. Educazione e prevenzione. La progettazione in ambito socio educativo. Trento: Centro Studi Erickson.

Flick, U. (2007). Introducción a la investigación cualitativa. Madrid: Morata.

Fraile Aranda, A. (Coord.). (2008). Los conflictos en las clases de educación física y algunas estrategias para la resolución. Barcelona: Graò.

Freire, P. (2004). Pedagogia dell'autonomia. Saperi necessari per la pratica educativa. Torino: EGA.

Galera, A. D. (2001). Manual de didáctica de la educación física. Vol. I. Barcelona: Paidós.

Goetz, J. P. y Lecompte, M. D. (1988). Etnografía y diseño cualitativo en investigación educativa. Madrid: Morata.

Goleman, D. (2008). Inteligencia emocional. Barcelona: Kairós.

González, J. \& Wagenaar, R. (2008). Universities' Contribution to the Bologna Process. Recuperado el 20 de noviembre de 2010 desde http://www.unideusto.org/tuningeu/home.html

Guba, E. G. (1981). Criterios de credibilidad en la investigación naturalista. En J. Gimeno Sacristán y A. Pérez Gómez (Eds.), La enseñanza: Su teoría y su práctica (pp. 148-165). Madrid: Akal.

Hellison, D. (2003). Teaching Responsibility through Physical Activity. $2^{\text {nd }}$ Ed. Champaign, IL: Human Kinetics.

Helliwell, J. F. (2002). How's Life? Combining Individual and National Variables to Explain Subjective Well-Being. NBER Working Paper, (9065). Recuperado el 13 de mayo de 2010 desde http://www.nber.org/papers/w9065

Hernández, J. S., Velázquez, R. y Alonso, D. (2004). La evaluación en Educación Física: Investigación y práctica en el ámbito escolar. Barcelona: Graò.

Hohmann, A., Lames, M. y Letzelter, M. (2005). Introducción a la ciencia del entrenamiento. Barcelona: Paidotribo.

Iori, V. (1996). Lo spazio vissuto. Luoghi educativi e soggettività. Firenze: La Nuova Italia.

Isidori, E. (2009a). La pedagogia dello sport. Roma: Carocci Editore. . (2009b). Outline of Sport Pedagogy. Roma: Aracne. 
e Fraile, A. (2008). Educazione, sport e valori. Roma: Aracne.

Jones, R., Armour, K. \& Potrac, P. (2004). Sports Coaching Cultures: From Practice to Theory. London: Routledge.

Larson, R. W. (2000). Towards a Psychology of Positive Youth Development. American Psychologist, vol.55 (1), 170-183.

Lev Pavlovich, M. (2001). Teoría general del entrenamiento deportivo. Barcelona: Paidotribo.

Malavasi, P. (2007). Pedagogia e formazione delle risorse umane. Milano: Vita e Pensiero.

Maslow, A. H. (1973). Motivazione e personalità. Roma: Astrolabio.

Maulini, C. (2013). Las competencias de los educadores deportivos para la promoción del bienestar en los jóvenes. Tesis doctoral. Valladolid: Universidad de Valladolid. (2012). L'allenatore-educatore nel positive youth development. In E. Isidori (Ed.), Pedagogia dell'allenamento (pp. 187-206). Roma: Edizioni Nuova Cultura.

(2011). Pedagogía del deporte y desarrollo del bienestar. En E. Isidori y A. Fraile Aranda (Coords.), La pedagogía del deporte hoy. Escenarios y desafíos (pp. 171-213). Roma: Edizioni Nuova Cultura. . (2006a). Pedagogia, benessere e sport. Roma: Aracne.

(2006b). Dalla Ricerca Azione (RA) all'Action Learning (AL). In S. Casucci e C.

Maulini (Eds.), Apprendere in età adulta. Ricerca e cambiamento dall'action learning al cooperative learning (pp. 11-52). Roma: Anicia.

Meghnagi, S. (2005). Il sapere professionale. Milano: Feltrinelli Editore.

Merico, M. (2004). Giovani e società. Roma: Carocci.

Miatto, E. (2012). Giovani verso il futuro. Per una pedagogia della transizione scuola-lavoro. Padova: Cleup.

Moreno, M. P. y Del Villar, F. (2004). El entrenador deportivo: Manual práctico para su desarrollo y formación. Barcelona: Inde.

Morin, E. (2000a). La testa ben fatta. Riforma dell'insegnamento e riforma del pensiero. Milano: Raffaello Cortina.

(2000b). I sette saperi necessari all'educazione del futuro. Milano: Raffaello Cortina.

(1993). Introduzione al pensiero complesso. Milano: Sperling \& Kupfer.

OCDE (2006). Definición y selección de competencias clave DESECO. Bruxelles: Parlamento Europeo.

Pascual Baños, C. (2011). La creación de un clima positivo en el aula de educación física: Una visión general. Tándem, (35), 61-67.

. (2010). El clima en el aula de Educación Física. En C. González Arévalo y T. Lleixa Arribas (Coords.), Didáctica de la educación física (pp. 47-64). Barcelona: Graó.

Patton, M. Q. (2001). Qualitative Research \& Evaluation Methods. Beverly Hills: Sage.

Petitpas, A. J., Cornelius, A. E., Van Raalte, J. L. \& Jones, T. (2005). A Framework for Planning Youth Sport Programs that Foster Psychosocial Development. The Sport Psychologist, vol.19 (1), 63-80.

Pietropolli, G. (2008). Fragile e spavaldo. Ritratto dell'adolescente di oggi. Roma-Bari: Laterza.

Prat, M. y Soler, S. (2003). Actitudes, valores y normas en la Educación Física y el deporte. Barcelona: Inde.

Processo di Bologna (2004). Descriptores di Dublino. Recuperado el 15 de mayo de 2012 desde www.jointquality.org

Rogers, C. R. (1983). Un modo di essere. Firenze: Psycho.

Rius, J. (2005). Metodología y técnica de atletismo. Barcelona: Paidotribo.

Schön, D. A. (1983). The Reflective Practitioner: How Professionals Think in Action. New York: Basic Books.

Thiess, G., Tschiene, P. y Nickel, H. (2004). Teoría y metodología de la competición deportiva. Barcelona: Paidotribo. 
UNESCO (2009). Conocimiento complejo y competencias educativas. IBE Working Papers on Curriculum Issues, (8), 1-13. Recuperado el 15 de mayo de 2012 desde http://www.ibe.unesco.org/ Weinberg, R. S. y Gould, D. (2010). Fundamentos de psicología del deporte y del ejercicio físico. Madrid: Editorial Médica Panamericana.

Weineck, J. (2005). Entrenamiento total. Barcelona: Paidotribo.

Zhelyazkov, T. (2001). Bases del entrenamiento deportivo. Barcelona: Paidotribo. 